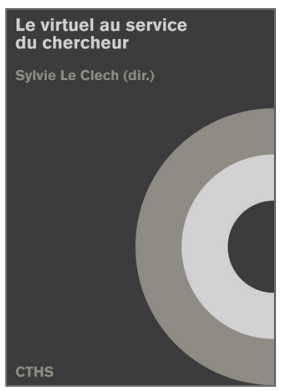

Sylvie Le Clech (dir.)

Le virtuel au service du chercheur

Éditions du Comité des travaux historiques et scientifiques

\title{
Le Moyen Âge reconstitué, entre réel et virtuel : une enquête au cœur de la fête médiévale
}

\section{Véronique Hochedé}

DOI : $10.4000 /$ books.cths. 15328

Éditeur : Éditions du Comité des travaux historiques et scientifiques Lieu d'édition : Éditions du Comité des travaux historiques et scientifiques Année d'édition : 2021

Date de mise en ligne : 21 juin 2021

Collection : Actes des congrès nationaux des sociétés historiques et scientifiques EAN électronique : 9782735509300

\section{Q boooks}

http://books.openedition.org

\section{Référence électronique}

HOCHEDÉ, Véronique. Le Moyen Âge reconstitué, entre réel et virtuel : une enquête au cœur de la fête médiévale In : Le virtuel au service du chercheur [en ligne]. Paris : Éditions du Comité des travaux historiques et scientifiques, 2021 (généré le 24 juin 2021). Disponible sur Internet : <http:// books.openedition.org/cths/15328>. ISBN : 9782735509300 . DOI : https://doi.org/10.4000/ books.cths. 15328 .

Ce document a été généré automatiquement le 24 juin 2021. 


\title{
Le Moyen Âge reconstitué, entre réel et virtuel : une enquête au cœur de la fête médiévale
}

\author{
Véronique Hochedé
}

1 La tradition de la fête médiévale est répandue dans les Hauts-de-France; depuis une vingtaine d'années, d'avril à octobre, des troupes de reconstitution en animent près d'une centaine par an ${ }^{1}$. Pour ces « reconstituteurs», il s'agit de présenter les modes de vie, civils ou militaires, d'un passé médiéval, et plus spécifiquement celui de la guerre de Cent $\mathrm{Ans}^{2}$. Les membres bénévoles de ces associations installent des campements le temps d'un week-end, animant des démonstrations de l'art de la guerre et de l'artisanat ${ }^{3}$. L'histoire vivante a pour but de rendre le passé «réel», de l'actualiser « comme si nous y étions». Le passé devient présent; on peut le voir, le toucher, le sentir et l'entendre. Les "reconstituteurs" mettent en scène le Moyen Âge qu'ils imaginent, en se référant à des sources historiques ${ }^{4}$. Ils établissent des cahiers des charges pour présenter des tableaux les plus fidèles possibles au passé. Une fois le cadre de la recréation défini, il reste à la mettre en œuvre ${ }^{5}$. Pour cela, ils s'emparent d'opportunités de la vie quotidienne qu'ils adaptent à leurs actualisations du passé. Toutefois, le réel oblige à de petits arrangements avec l'image du passé qu'on a en tête, au prix de certaines entorses au principe d'authenticité. Parfois même, il empêche totalement l'actualisation. Ainsi le jeu du «reconstituteur » consiste en des allées et venues permanentes entre réel et virtuel, entre ce qu'il a en tête, qu'il veut rendre vivant, et les arrangements que le quotidien l'oblige à adopter dans cette projection du passé.

2 Cet article s'appuie sur les observations effectuées lors d'une trentaine de fêtes visitées dans les Hauts-de-France, entre 2015 et 2019, dans le cadre d'une thèse en anthropologie en préparation et sur celles menées au sein d'une troupe que j'ai intégrée ${ }^{6}$. Cette association, spécialisée dans le XIV siècle, propose la recomposition d'une rue artisanale, chacun des membres incarnant un artisan ou un commerçant. 


\section{Recomposer un passé médiéval au XXI ${ }^{\mathrm{e}}$ siècle}

3 Être «reconstituteur ", c'est se choisir un personnage et lui donner vie pendant une fête médiévale? . Il faut adopter un patronyme d'époque, se doter d'un costume et se composer un rôle pour lequel une formation est nécessaire. En effet, l'individu doit être en capacité de mener des interactions avec le public par le biais de démonstrations de gestes ancestraux ${ }^{8}$. Dans la troupe, nous sommes six membres actifs. Marie, la cinquantaine, est la caution historique pour définir les contours de ce que doit être notre recomposition d'un passé médiéval. Elle travaille dans le secteur de l'aide à la personne, se dit passionnée d'histoire depuis enfant. Elle s'est formée en autodidacte sur le Moyen Âge. Elle possède un fonds de livres, enrichi année après année, dont elle parle avec fierté ; c'est même une des premières choses qu'elle m'ait dites alors que je ne faisais pas encore partie de la troupe. Effectivement, dans sa bibliothèque assez fournie se trouve une diversité de livres: des écrits d'historiens, des ouvrages sur le costume, sur l'alimentation, mais aussi des récits romancés sur les $\mathrm{XIV}^{\mathrm{e}}$ et $\mathrm{XV}^{\mathrm{e}}$ siècles. Elle a visité bon nombre de musées, de châteaux et d'édifices religieux où se trouvent des gisants médiévaux. Ainsi, elle s'est construit au fil du temps une culture personnelle sur le Moyen Âge, mêlant faits historiques et sociaux, réels ou fictifs - ce qui lui permet de composer son personnage et de lui donner la parole dans différentes situations. Marie estime la durée de sa formation à une dizaine d'années, mais reste toujours à l'affût de nouvelles informations. C'est donc sur la base de ces savoirs, constitués en autodidacte parmi une variété de sources hétérogènes, qu'est établie la ligne directrice suivie par l'ensemble de la troupe. C'est donc vers elle que je me tourne quand j'ai besoin d'informations à propos de mon costume ou de mon rôle de femme de commerçant. Mais si les connaissances permettent de poser le cadre de ce qui sera mis en scène, encore faut-il pouvoir le composer avec les moyens contemporains à disposition'. Où trouve-t-on les objets "authentiques" pour figurer ceux du Moyen Âge?

C'est en compagnie de Marie et d'autres membres de l'association que je visite le Marché de l'histoire de Margny-lès-Compiègne ${ }^{10}$. Il est hors de question de manquer cet événement semestriel où sont vendus des accessoires neufs spécifiques à la reconstitution, allant de l'épingle à cheveux jusqu'à l'armure. Il est possible de s'y équiper dans tous les domaines - habillement, alimentation, équipement de guerre, tente... - et toutes les époques (fig. 1). 
Fig. 1 - Stand au Marché de l'histoire, Margny-lès-Compiègne (Oise).

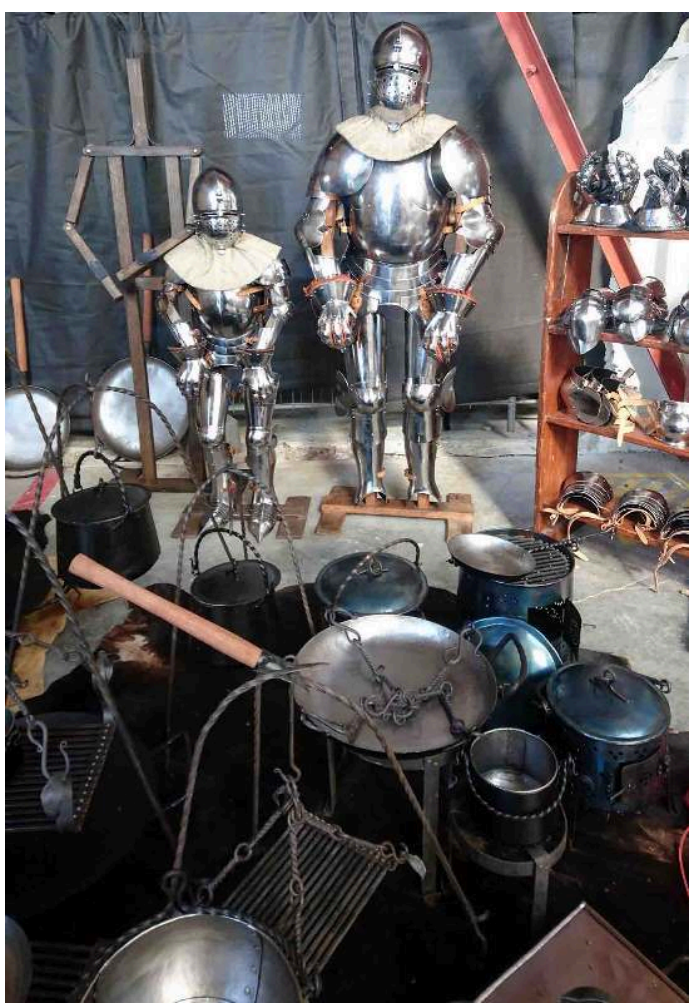

(c) V. Hochedé.

5 Les membres de la troupe visitent le marché munis d'une liste d'achats établie par avance : il faut renouveler du matériel perdu ou usé. L'objectif est de se procurer de nouveaux ustensiles de cuisine pour faciliter la tâche d'Adèle - cuisinière sur les camps -, à savoir une poêle et une marmite en fer. Tout ce qui constitue le gros équipement de l'association y a été acquis au fil des ans. Ce sont par exemple les six tentes et l'auvent, les deux bancs, des coffres en bois et du matériel de cuisson (bac à feu, trépied). Une fois les achats effectués, la troupe se promène dans les allées du marché. L'événement est prisé par les « reconstituteurs » et on s'y retrouve entre pairs. On ne perd pas non plus de vue qu'il peut y avoir des opportunités chez les commerçants. Adèle s'est longtemps attardée devant les pichets en grès d'un artisan, mais aucun ne trouve grâce à ses yeux parmi les nombreux modèles, reproductions de pièces anciennes rangées par époque. Moi-même, ne disposant pas de verre pour la reconstitution, je regarde les gobelets en céramique mais n'achète rien. Chacun des membres de la troupe possède sa propre vaisselle et lors des premières sorties, on m'a prêté le nécessaire. Adèle m'avait alors conseillé de me procurer un verre par un autre biais que celui de l'achat auprès d'un marchand spécialisé : "En faisant tes courses alimentaires, prends un pot de fromage de brebis. Une fois lavé, il te servira de verre. »

6 Au sein de cette troupe, l'achat de produits neufs en dehors du gros équipement pour le jeu de reconstitution n'est effectué que sur des articles spécifiques. C'est par exemple le cas avec des aiguillettes de laçage et des boutons. Ces derniers, bombés et en étain, sont à coudre le long des manches et sur le devant de ma robe. Ils ne servent en rien à la fermeture du vêtement : "C'est signe de richesse ", me dit Marie. Dans mon rôle de femme d'artisan, il faudra que j'envisage d'en acheter une cinquantaine; vendus un euro pièce. Il est difficile de se les procurer ailleurs que chez un marchand spécialisé 
dans la reconstitution. Marie distingue immédiatement ceux dont la forme et le motif conviennent pour le XIV ${ }^{e}$ siècle parmi les nombreux modèles exposés dans des casiers. C'est différent pour les boutons en bois, qu'Adèle préconise de fabriquer soi-même. Elle s'en est confectionnés elle-même dans du buis, qu'elle a taillé en rondelles plus ou moins uniformes et percé de deux trous. Elle tient tour à tour les rôles de cuisinière, de servante et parfois celui de fileuse de laine. Mon statut dans le jeu est autre: je dois montrer ma richesse par des accessoires luxueux. Sur un stand de bijoux, Marie me montre les pierres compatibles pour la reconstitution de l'époque mais aussi du rang social. Comme pour les boutons, je dois respecter certains critères. Ce ne sont pas des accessoires de pacotille, mais de véritables bijoux de valeur qui me sont proposés. Le coût de l'équipement me semble onéreux, mais Marie me rassure : «Tu t'équiperas au fur et à mesure. Pour les bijoux, tu peux te les faire offrir en cadeaux à Noël ou ton anniversaire. " C'est ce qu'elle a fait elle-même pour sa cuillère en étain, son porteaiguilles ou encore le petit flacon de parfum monté en broche et qu'elle porte sur sa tenue. Ce sont de belles pièces d'exception, pour lesquelles elle n'a pas hésité à faire appel au savoir-faire d'artisans et d'orfèvres spécialisés dans l'époque médiévale.

7 Cette visite permet d'évaluer tout ce qu'il manque pour équiper mon stand et étoffer mon personnage: des coffres en bois, des accessoires vestimentaires, des objets nécessaires pour l'activité de commerçant, mais aussi décoratifs. Tenir le rôle d'un marchand du Moyen Âge a un coût et la richesse du personnage créé dépend de l'investissement financier consenti dans la vie réelle. Marie me souffle qu'il y a d'autres alternatives pour se procurer le matériel nécessaire et me propose de visiter les solderies et les brocantes dans lesquelles je pourrais trouver des éléments moins onéreux à intégrer au nombre de mes artéfacts. Elle me conseille d'ailleurs de garder sur moi un échantillon de tissu au cas où j'en trouverais par hasard à un prix intéressant. Elle m'explique qu'il faut toujours être à l'affût de ce qui pourrait être investi dans le jeu, tel quel ou transformé. C'est le cas dans une brocante, avec des pots à épices en bois surmontés d'un couvercle et pourvus d'une fine plaque métallique indiquant l'ancien contenu. Marie me conseille de les acheter: une fois l'étiquette retirée, ils seront intégrés dans mon échoppe. Plus loin, je la vois s'arrêter devant un manteau de fourrure. Je m'étonne de l'intérêt qu'elle porte à ce vêtement désuet, qui, de plus, ne doit pas être à sa taille. Elle m'explique qu'en découpant des bandes, elle pourra les coudre aux manches et au col de sa robe, en signe de richesse. Au Marché de l'histoire, Marie avait en effet délaissé les chutes de fourrure, estimant qu'elle pourrait en trouver ailleurs à un meilleur rapport qualité-prix. C'est chose faite dans cette brocante : ce vêtement d'occasion lui permettra d'agrémenter d'autres tenues. Mais au Marché de l'histoire, Marie avait aussi choisi deux morceaux de cuir assez semblables et m'avait suggéré de m'en faire des chaussures - me proposant d'ailleurs de me mettre en contact avec un "reconstituteur» qui pourrait confectionner une paire à ma mesure.

8 Pour construire les mises en scène, on pioche dans différentes ressources et on assemble des éléments hétéroclites qui constitueront une unité sur le camp. Les objets façonnés par des artisans sont préférables, en ce qu'ils correspondent à une représentation partagée dans la pratique de ce qu'ils devaient être au Moyen Âge ; ils répondent à la norme. Mais quand on ne dispose pas de moyens, il faut trouver des artéfacts par un autre biais ou les fabriquer soi-même. Le premier écueil est de présenter des objets dont l'anachronisme serait flagrant. Ce serait apporter du discrédit à sa pratique de reconstitution, qui par définition doit être fidèle à la réalité historique. 
Ainsi, un grand soin est apporté au visuel proposé, en gommant les traces d'une modernité trop visibles. Toutes les matières que l'on pourrait qualifier d'anciennes trouvent grâce aux yeux des « reconstituteurs" - coton, lin, verre, bois... -, car déjà utilisées au Moyen Âge. D'autres sont totalement écartées : celles dérivées du pétrole, en désaccord avec l'image de la société qu'on cherche à reproduire, bien antérieure à l'ère industrielle. Peu importe la provenance des objets, leur origine et leur fonction : s'ils présentent une cohérence avec la représentation que se fait le "reconstituteur " d'un objet "assez ancien ", ils sont intégrés dans le jeu. Ainsi, le pilon en bois, déniché sur une brocante, peut intégrer le comptoir de mon échoppe - pour peu que je retire l'étiquette autocollante figurant une ville touristique. En effet, il est en bois et c'est un ustensile dont on sait qu'il était fait usage à l'époque : peu importe sa forme moderne et le fait qu'il ait été produit de façon mécanique. Il suffira de l'utiliser devant le public et d'appuyer la démonstration d'un discours décrivant l'usage médiéval. L'objet dépossédé d'une valeur marchande retrouve une utilité : il enrichit la scène médiévale. Mais plus encore : intégré dans la recomposition, il se voit conférer une valeur symbolique de preuve, de témoignages d'un mode de vie passé, avec lequel il n'a pourtant aucun lien ${ }^{11}$.

Si la projection d'objets contemporains dans les mises en scène du passé semble relativement simple, qu'en est-il de celle des individus? Pour animer ces présentations du Moyen Âge, ces derniers doivent composer des personnages. Comment le font-ils? Jusqu'où peut-on aller dans l'immersion médiévale ? Comment le quotidien est-il mis à contribution pour alimenter le jeu?

\section{Vivre au Moyen Âge au quotidien}

10 Le mot «incarner » est employé par les membres de ces associations dans le sens d'endosser l'identité d'une personne qui a vécu dans le passé - généralement attesté par une simple mention dans un manuscrit médiéval - ou de celle qui aurait pu vivre. Pendant la fête, l'identité civile du quotidien est mise de côté au profit de celle de la reconstitution. Il est difficile d'ailleurs de discerner quand se situe la fin de la projection tant l'individu parait plongé en permanence dans le rôle médiéval. Dans certaines situations, l'identité de reconstitution semble prendre le pas sur celle de la vie réelle. Dans la troupe, on se nomme uniquement par le nom de son personnage reconstitué et cela même en dehors des activités médiévales - échanges de mails, conversations téléphoniques, rencontres physiques. Ainsi, je n'ai connu le véritable nom de l'une d'entre nous que plusieurs mois après mon entrée dans la troupe. Plus qu'un rôle de composition qui durerait le seul temps de la fête, l'identité de reconstitution est utilisée hors cadre : doit-on en déduire qu'elle serait préférée à celle assignée dans le civil ? C'est d'ailleurs bien plus que le nom qui est exprimé dans le fait de nommer l'autre: le rang est aussi énoncé puisque pour les femmes, le substantif «dame » est apposé à l'appellation. C'est être un autre par le nom, mais aussi par le statut social.

11 L'enchevêtrement des identités ne se limite pas à la nomination. La recomposition gagne de la valeur avec les expériences menées en son titre au quotidien ${ }^{12}$. Marie m'encourage par exemple à adopter certaines attitudes qui amélioreront, selon elle, la reconstitution. Le costume est essentiel mais il ne suffit pas pour rejouer le Moyen Âge : la manière de le porter est également importante. Pour cela, elle me conseille de me vêtir en robe le plus souvent possible. Elle-même s'habille à son domicile avec de 
longues robes de diverses origines - tenue estivale, boubou africain, caftan. Elle m'explique que les filles d'aujourd'hui, «toujours en pantalon ", ont une démarche qui n'est pas celle des femmes du Moyen Âge: "Elles font des enjambées d'homme", déplore-t-elle. Elle me conseille donc de porter des tenues vestimentaires qu'elle juge approchant de celles de la femme médiévale, afin que mon corps adopte un port « convenable». Il s'agit d'incorporer ce qui serait une démarche féminine tout droit héritée du Moyen Âge, ou du moins d'une époque où la femme ne portait pas de pantalon. À cette hexis corporelle, il faut ajouter l'adoption de caractéristiques physiques. Marie a elle-même les cheveux longs et me préconise de ne pas couper les miens, car la femme « respectable » du XIV ${ }^{e}$ siècle ne le faisait pas, mais aussi parce que certaines coiffures médiévales nécessitent une longueur suffisante pour être réalisées, comme un chignon ou des "oreillettes" - bien qu'un voile vienne finalement les masquer. Cela laisse supposer que si on veut proposer la meilleure reconstitution possible, la plus fidèle aux modes de vie ancestrale, il faut non seulement que la projection soit tenue de façon quasiment permanente dans la vie quotidienne, mais soit aussi inscrite dans le corps même.

Pour Marie et Adèle, les plus investies de la troupe, s'impliquer dans des expérimentations en dehors des fêtes fait partie de la manière de vivre la reconstitution. Le temps libre est mis à contribution pour tester des techniques utilisées au Moyen Âge. Adèle tente par exemple de reproduire des recettes de cuisine médiévales, s'appliquant à ne cuisiner que les ingrédients en usage à l'époque. Ainsi, elle apporte sur le camp un bocal d'œufs au vinaigre qu'elle a réalisé l'hiver précédent. Marie, quant à elle, passe de longues heures à coudre à la main une robe vue sur une iconographie. Elle cherche à comprendre la construction de cette robe de grossesse. Elle coud ainsi un vêtement semblable à ce qu'elle voit sur la reproduction, et invente ce que l'image ne montre pas grâce à ses compétences de couturière et ses connaissances en matière de maternité à l'époque médiévale. Un autre jour, elle présente une robe de couleur jaune dont elle est fière : elle a teinté cette pièce en plusieurs étapes, jusqu'à obtenir exactement le même coloris que celui de la photocopie. Si elle n'exige pas que nos tissus soient teints maison, elle insiste en revanche pour que nos tenues soient cousues à la main, en suivant certains patrons, certains points et en utilisant du coton ou du lin. Elle m'a montré des aiguilles en os au Marché de l'histoire, du même modèle que celles utilisées au XIV siècle. Adèle en a d'ailleurs déjà confectionné avec des os de lapin. Si la fabrication des aiguilles a été un succès, il en est autrement de leur usage : elles cassent très facilement. Adèle a ainsi renoncé à utiliser ces aiguilles dont il aurait fallu constituer un stock important pour être en mesure de coudre ne serait-ce qu'une seule pièce. Elle adopte alors les métalliques à chas plat, elles-mêmes dites "historiques". Mais elle prend soin d'exposer les aiguilles en os auprès de son matériel de fileuse. Pour Marie et Adèle, l'expérimentation est indissociable de la pratique de reconstitution: vivre le Moyen Âge ne prend pas fin après la fête médiévale. Elles explorent ce passé en testant les gestes ancestraux. Ces expériences sont autant de preuves attestant de la faisabilité des techniques médiévales et qui apportent une valeur supplémentaire aux mises en scène. Elles n'hésitent pas à expliquer au public comment elles ont reproduit ces gestes, démontrant ainsi qu'ils furent effectués tels qu'elles les décrivent, puisqu'elles sont parvenues à un résultat similaire. Avec toute cette phase d'expérimentations et d'épreuves personnelles, la projection dépasse le cadre du temps de reconstitution. C'est en permanence que cela se joue : on pense toujours à ce passé à mettre en place, 
combinant vie quotidienne avec reconstitution. Cet encastrement voulu est-il toujours réalisable? D'ailleurs, le monde réel ne s'invite-t-il pas lui aussi sur la scène de la recomposition? On met en place un cadre et on tente d'en garder la maîtrise ; mais estce toujours possible?

\section{De la difficulté à vivre le passé aujourd'hui}

13 Les fêtes se tiennent le week-end. Pour participer, il faut généralement postuler en répondant à des appels à candidatures, diffusés parfois jusqu'à six mois avant la date de l'événement. Or, il est difficile de certifier que l'on sera disponible à ce moment-là quand on travaille par exemple dans les secteurs de l'aide à la personne ou du commerce. Les jours de congés demandés ne sont pas toujours accordés à l'approche de l'échéance - Marie en a fait l'amère expérience, à plusieurs reprises. Pour le « reconstituteur », participer à une fête signifie le plus souvent poser une demi-journée précédant le week-end. Transporter l'équipement nécessite de louer un véhicule adapté ; puis il faut charger le matériel avant de prendre la route, pour effectuer parfois des centaines de kilomètres. Arrivé sur les lieux, l'installation du camp peut prendre deux à trois heures, selon le nombre de membres présents. Il faut aussi disposer de temps après le week-end pour effectuer les opérations inverses : trajet de retour, rangement du matériel, restitution du camion. Bernard, l'époux de Marie, explique qu'il lui faut à peu près une semaine pour se remettre physiquement de la fatigue occasionnée par une sortie médiévale. Une ancienne "reconstitutrice» m'explique que ce travail annexe l'a incitée à quitter la pratique : «Je passais une partie de mon temps libre à faire les courses pour les repas, préparer tout le nécessaire pour le couchage et les costumes pour mon mari, nos trois enfants et moi-même. Et au retour, il fallait laver le linge, ranger, tout cela en plus de mon activité professionnelle. » Elle participe toujours aux fêtes médiévales avec son époux, mais au sein d'une troupe musicale.

14 Malgré tous les efforts engagés à recomposer le passé, la projection ne fonctionne pas toujours. En effet, actualiser le passé en gardant pour ligne directrice le respect des usages médiévaux rencontre des limites. Par exemple, la nourriture des repas du weekend est stockée dans des glacières posées dans la tente-cuisine, hors de la vue du public. Il n'est pas question de quitter le campement pendant la durée de la fête dans cette immersion continue, il faut donc la conserver sur place. Adèle a apporté entre autres aliments un faisan qu'elle conserve grâce à des accumulateurs de froid. Ce gibier lui donnera l'opportunité de proposer une belle animation en plus du repas, puisqu'elle envisage de plumer la bête en public. Bien sûr, elle la cuisinera avec une recette que nous pourrons apprécier : il est impossible de reconstituer le goût de l'époque qui, de plus, ne doit pas correspondre à nos attentes actuelles en matière culinaire. Mais le public verra sur la table un gibier et les légumes déjà servis au XIV siècle. Malheureusement, à l'ouverture de la glacière le samedi matin, Adèle se rend compte que la viande a tourné. Animation et repas de gibier sont dès lors impossibles, et il faut trouver des solutions pour remplir les estomacs. Elle décide de décaler l'ordre des repas, supprimant celui du dimanche soir. C'est celui qui devait être pris avant la route du retour, juste après avoir remballé le matériel. Il n’a rien de médiéval - pain, jambon et chips - mais présente l'avantage d'être pratique : pas de cuisson et une conservation simple quand tous les dispositifs pour maintenir le froid ne sont plus actifs. Ce repas de 
substitution est servi le soir même, à l'abri du regard du public. Le recours aux facilités contemporaines peut advenir pour peu que cela ne soit pas montré ; le feu est allumé avec un briquet à gaz, mais Adèle garde un modèle ancien en poche pour le présenter au public. Les bougies allumées le soir proviennent du supermarché mais d'autres, achetées chez un artisan cirier, sont posées en évidence sur la table en journée. De même, du café est servi le matin : on ne saurait se passer de cette boisson qui n'était pourtant pas consommée à l'époque. Si par hasard on venait à me poser des questions, Adèle me conseille de présenter la boisson comme de l'épeautre. On compose donc avec les deux mondes, réel et virtuel, masquant toujours les traces de ce réel indésirable. Que la reconstitution déborde sur la vie quotidienne ne pose pas de souci. Mais à l'inverse, que le monde moderne fasse des intrusions dans la recomposition est beaucoup moins supportable.

On investit beaucoup, en temps ou financièrement, pour construire la mise en scène. Si la qualité visuelle est assurée dans une maîtrise du cadre de jeu, et si l'expérimentation et parfois une incorporation sont effectuées pour approcher le plus possible du modèle à reproduire, ces projections proposent-elles pour autant des représentations fidèles au passé, tel que le suggère le principe d'authenticité recherchée par l'histoire vivante ${ }^{13}$ ? L'observation menée lors d'une trentaine de fêtes ne manque pas de poser question à ce sujet. Devant la volonté affichée d'être respectueux de la vérité historique, on ne peut qu'être étonné de la répartition des rôles opérée. Dès mon entrée dans la troupe, Marie me demande d'acheter un chapelet. Dans une recomposition d'une femme du $\mathrm{xIV}^{\mathrm{e}}$ siècle, il est impensable de ne pas porter de signes religieux. Elle me montre aussi des pendentifs compatibles avec l'époque. Mon costume de femme d'artisan comprend donc un pendentif porté de façon visible au-dessus de ma robe et un chapelet fixé à la ceinture. Je m'en étonne, car je vois peu de «reconstitutrices » qui en portent... et encore moins d'hommes en affichant sur leur costume. En effet, dans cette reconstitution médiévale, religieux et paysans sont peu présents alors qu'ils composaient la majeure partie de la société médiévale, comme en attestent des sources historiques. Si la religion est bien évoquée à travers des éléments décoratifs iconographies, autels à la gloire de saints ou encore mises en scène de reliques -, elle est peu incarnée dans des rôles de religieux. Et quand elle l'est, c'est le plus souvent sous une forme caricaturale: le bon vivant, le moine défroqué, celui qui fait des blagues. Le rôle n'est pas tenu au sérieux comme pour les autres catégories que sont les artisans ou les combattants. Bien sûr, il existe dans certaines fêtes un "reconstituteur " d'un pèlerin de Saint-Jacques de Compostelle et celui d'un prêtre bénissant les combattants, mais c'est peu au regard des autres personnages (fig. 2). 
Fig. 2 - « Reconstituteur » d'un pèlerin.

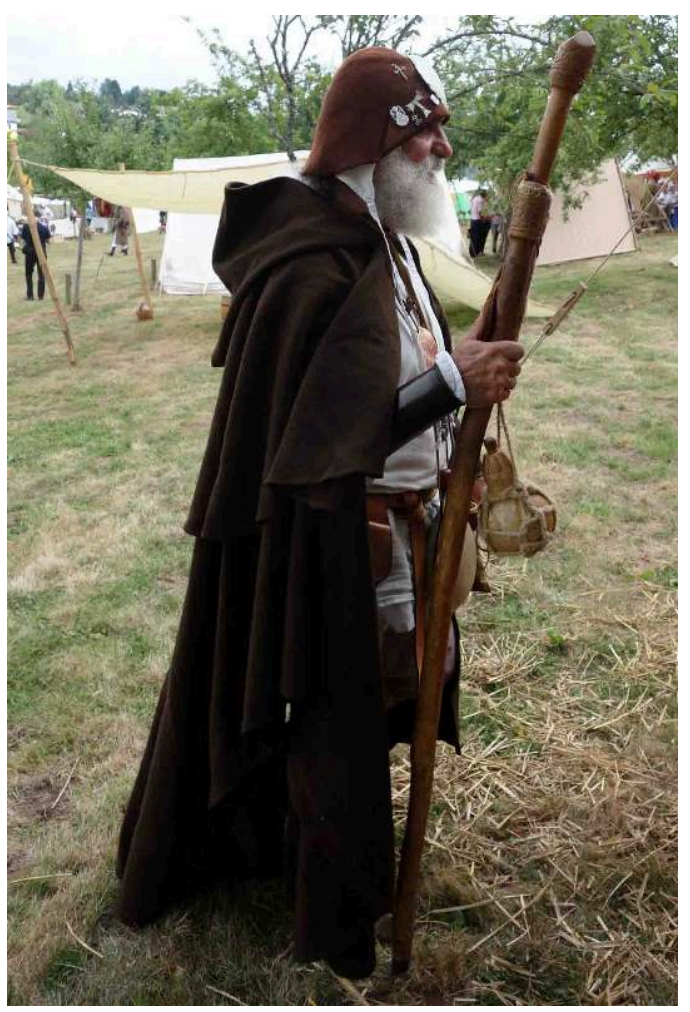

(c) V. Hochedé.

16 Quand j'interroge Marie à ce sujet, elle déplore cet état de fait. Elle a cousu une bure de moine, au cas où un nouveau membre masculin entrerait dans la troupe. Cela lui permettrait de participer aux fêtes immédiatement en attendant de pouvoir tenir un rôle d'artisan. C'est ce même type de réponse que j'ai reçu de quelques membres d'une troupe, présentant des moines facétieux lors d'une mise en scène de combat, proposant à boire de l'eau salée aux soldats. Quelques membres d'une troupe de moines facétieux, proposant de l'eau salée à boire aux soldats lors d'une mise en scène de combat, m'expliquent aussi que c'est le moyen pour eux de participer à l'événement sans encore posséder de costumes adéquats. Le religieux est donc perçu comme un personnage de transition.

17 Si bien des valeurs du Moyen Âge sont louées sur les camps médiévaux, la religion - qui semble pourtant avoir été très présente dans la société médiévale, dictant le rythme même des principaux actes de la vie - n'en fait pas partie. Au nombre des efforts consentis, que ce soit dans la vie de tous les jours ou au sein des campements, à la mise en scène du passé médiéval qui serait la plus « histo " possible, cette absence surprend. Cette projection semble impossible à mettre en œuvre. Celui qui endosse le rôle d'un personnage médiéval opère des choix dans ce qu'il estime être acceptable de reproduire et ce qui ne l'est pas, parfois au détriment du principe de l'authenticité.

La pratique de reconstitution demande un engagement qui s'avère parfois difficile à tenir. Le souci de la meilleure recomposition peut devenir obsessionnel, accaparant l'individu jusque dans les actes de sa vie quotidienne. Certains pratiquants cessent la reconstitution, devenue insupportable : trop d'argent, de temps, d'énergie à consacrer 
à cette activité de loisir. Parfois, cela demande aussi de recomposer des valeurs qu'on ne partage pas.

Pour d'autres, à l'inverse, il serait difficile de se passer de cette projection, si prenante soit-elle. Au contraire, pour eux, ce n'est jamais trop ! Le temps de la fête ne suffit pas, on ne vit jamais assez ce passé dans lequel on se donne pour mission de présenter le "véritable » Moyen Âge, loin des clichés et des représentations négatives, en exhumant le «meilleur » de l'époque pour en faire un tableau élogieux. Il est à se demander si ce monde dans lequel on se projette n'est pas préférable à celui de la vie quotidienne, d'autant plus qu'on s'y compose un rôle sur mesure.

\section{BIBLIOGRAPHIE}

BARTHOLEYNS Gil, «Le passé sans l'histoire : vers une anthropologie culturelle du temps », Itinéraires : littérature, textes, cultures, $\mathrm{n}^{\circ}$ 3, 2010, p. 47-60.

BEAUD Stephane et WEBER Florence, Guide de l'enquête de terrain : produire et analyser des données ethnographiques, 4 e éd., Paris, La Découverte, 2010.

BECKER Howard S., «Sur le concept d'engagement », SociologieS, éd. numérique, 2006. [URL :

http://journals.openedition.org/sociologies/642]

BENSA Alban et FABRE Daniel, Une histoire à soi : figurations du passé et localité, Paris, Éditions de la Maison des sciences de l'homme, 2001.

BERLINER David, « Anthropologie et transmission », Terrain : anthropologie \& sciences humaines, éd. numérique, $\mathrm{n}^{\circ}$ 55, 2010. [URL : http://terrain.revues.org/14035]

BOLTANSKI Luc et ESQUERRE Arnaud, Enrichissement : une critique de la marchandise, Paris, Gallimard, 2017.

COHEN Claudine, « Faux et authenticité en préhistoire », Terrain : anthropologie \& sciences humaines, éd. numérique, $\mathrm{n}^{\circ} 33,1999$. [URL : http://terrain.revues.org/2685]

CRIVELLO Maryline, « Comment on revit l'histoire : sur les reconstitutions historiques

(1976-2000) », La Pensée de midi, vol. III, n³ 3, 2000, p. 69-74.

CRIVELLO Maryline, « Du passé, faisons un spectacle ! Généalogies des reconstitutions historiques de Salon et Grans en provence ( $\mathrm{XIX}^{\mathrm{e}}-\mathrm{XX}^{\mathrm{e}}$ siècles) », Sociétés \& Représentations, vol. XII, $\mathrm{n}^{\circ} 2,2001$, p. 225-234.

FABRE Daniel, «Le retour des héros », Hypothèses, vol. V, n 1, 2002, p. 225-228. [URL : https:// www.cairn.info/revue-hypotheses-2002-1-page-225.htm]

GLEVAREC Hervé et SAEZ Guy, Le patrimoine saisi par les associations, Paris, La Documentation française, 2002.

PETERSON Richard A., « La fabrication de l'authenticité : la country music », Actes de la recherche en sciences sociales, vol. XCIII, L'invention du passé national/Le ghetto vu de l'intérieur, 1992, p. 3-20. 
TUAILLON DEMÉSY Audrey, «L'histoire vivante médiévale : pour une ethnographie du "passé contemporain" ", Ethnologie française, vol. XLIV, n 4 , 2014, p. 725-736.

TUAILLON DEMÉSY Audrey, La re-création du passé : enjeux identitaires et mémoriels. Approche socioanthropologique de l'histoire vivante médiévale, Besançon, Presses universitaires de Franche-Comté, 2013.

\section{NOTES}

1. H. Glevarec et G. Saez, Le patrimoine saisi par les associations.

2. D. Fabre, « Le retour des héros ». Voir aussi A. Bensa et D. Fabre, Une histoire à soi [...].

3. A. Tuaillon Demesy, La re-création $d u$ passé $[. .$.$] ; Id., "L'histoire vivante$ médiévale $[. .]$.$» .$

4. M. Crivello, « Du passé, faisons un spectacle ! [...]».

5. Id., « Comment on revit l'histoire [...]», p. 74.

6. S. Beaud et F. Weber, Guide de l'enquête de terrain [...].

7. Il existe d'autres lieux pour la reconstitution historique, notamment hors public.

8. D. Berliner, « Anthropologie et transmission ».

9. R. A. Peterson, « La fabrication de l'authenticité [...]».

10. Événement organisé par l'APHV, marché de produits pour la reconstitution à Margny-lès-Compiègne (Oise).

11. L. Boltanski et A. Esquerre, Enrichissement [...], p. 71.

12. C. Cohen, «Faux et authenticité en préhistoire ». Voir aussi H. S. Becker, «Sur le concept d'engagement ».

13. G. Bartholeyns, « Le passé sans l'histoire [...]».

\section{RÉSUMÉS}

Depuis une vingtaine d'années, des troupes de reconstitution animent des fêtes médiévales dans les Hauts-de-France. Elles proposent des mises en scène du Moyen Âge, élaborées en vue de favoriser l'immersion du public dans le passé. Cela est rendu possible au prix de l'investissement personnel $\mathrm{du}$ "reconstituteur»: il lui faut définir ce qui sera mis en scène, effectuer des recherches sur l'époque, se doter des artéfacts nécessaires pour livrer le tableau vivant de ce qui a pu exister au XIV siècle, de la manière la plus authentique possible. Pour les plus passionnés, la reconstitution médiévale peut devenir une préoccupation de tous les instants, dans un souci d'améliorer la qualité de la projection. C'est dans un mouvement perpétuel entre l'actualisation du passé tel qu'il est imaginé et le réel de la vie quotidienne que s'élabore la recomposition. Jusqu'où est prêt à s'investir le « reconstituteur »? Quelles sont les limites? 
AUTEUR

VÉRONIQUE HOCHEDÉ

Doctorante en anthropologie, Centre universitaire de recherches administratives et politiques de Picardie (CURAPP, UMR 7319), université de Picardie - Jules-Verne 